\title{
Combined reflectance and Raman spectroscopy to assess degree of in vivo angiogenesis after tissue injury
}

\author{
Shailesh Agarwal, \\ Hwang $^{a}$, Michael D. Morris, ${ }^{b}$ Benjamin Levi ${ }^{a}$ \\ ${ }^{a}$ University of Michigan, Department of Surgery, 1500 E. Medical Center Dr, Ann Arbor, MI, 48109 \\ ${ }^{\mathrm{b}}$ University of Michigan, Department of Chemistry, 930 N. University Avenue, Ann Arbor, MI, 48109 \\ *Authors contributed equally to this work.
}

Address all correspondence to: Michael D. Morris, E-mail: mdmorris@umich.edu or Benjamin Levi, E-mail: blevi@umich.edu, phone number: (847)571-6511

Short title: Reflectance spectroscopy to assess angiogenesis

\section{Author Contributions}

S.A., W.R.L., and B.L. designed the experiments. S.A., and M.T.C. performed all murine traumas; S.J.L., M.T.C., C.H. collected data; M.D.M. and B.L. helped with study design and analysis; M.D.M. and B.L. reviewed data and edited manuscript; S.A., W.R.L., M.T.C., and C.H. drafted the manuscript.

\begin{abstract}
Background: Angiogenesis, the formation of blood vessels, is a critical aspect of wound healing. Disorders of wound healing are often characterized by lack of angiogenesis, a condition frequently observed in aging and diabetic patients. Current techniques for assessing blood at injury sites are limited to contrast-imaging, including angiography. However, these techniques do not directly observe oxygenation of blood and are not amenable to serial evaluation. A multi-modal non-invasive reflectance and Raman spectrometer has been proposed to help clinicians as a point-of-care (POC) tool to interrogate local angiogenesis and tissue architecture, respectively. The spectrometer system is a rapid, non-invasive, and label-free technology well-suited for the clinical environment. Materials and Methods: To demonstrate feasibility, the spectrometer system was employed to interrogate angiogenesis serially over 9 weeks as a result of heterotopic ossification (HO) development in a validated murine model. End-stage HO was confirmed by micro computed tomography (micro-CT).

Results: Our preliminary results suggest that reflectance spectroscopy can be used to delineate vessel formation, and that pathologic wounds may be characterized by unique spectra. In our model, HO formed at sites 1-3, while sites 4 and 5 did not have radiographic evidence of $\mathrm{HO}$.

Conclusions: A POC system like that demonstrated here shows potential as a non-invasive tool to assess local angiogenesis and tissue architecture that may allow for timely intervention in a clinical setting.
\end{abstract}

Keywords: Raman spectroscopy; reflectance spectroscopy, near-infrared light; light scatter; tissues; angiogenesis

\section{Disclosures}

The authors have no conflicts of interest to disclose. 


\section{Introduction}

Heterotopic ossification (HO) is a condition of pathologic wound healing in which patients develop ectopic osseous lesions after trauma. (1-3) Patients at risk for HO include those with large surface-area burns and severe musculoskeletal injury. These patients are exposed to massive inflammatory insults, which lead to pathologic cellular differentiation, cartilage formation, and ossification. These ectopic lesions are often painful, can lead to new wounds, inhibit joint motion, and may require surgical excision of tissue. After surgical excision, patients are at risk for recurrence due to the resurgence of local inflammation.(4) Furthermore, there exists no early detection or adequate treatment options today for a process closely associated with dynamic changes in local vasculature.

Soft tissue wound healing is another commonly encountered clinical picture intimately reliant on vascular adaptation. Although early treatment would be beneficial to prevent the chronic effects associated with poor angiogenesis, diagnostic methods are limited.(5, 6) Current techniques for real-time vascular imaging are limited to contrast-based techniques including vascular angiography.(6, 7) These techniques are often invasive, resource-intensive, and impractical for serial imaging. Noninvasive spectroscopy, including reflectance and Raman spectroscopy, has emerged as a tool to image vessels at the bedside.(8) Reflectance spectroscopy assesses oxygenated and de-oxygenated hemoglobin present in local vessels due to variations in the absorption spectra of the respective forms of hemoglobin. Raman spectroscopy assesses the chemical fingerprint of a sample. Clinical applications of reflectance spectroscopy include analyzing malignant tissue(9) and tissue oxygenation.(10, 11) Clinical applications of Raman spectroscopy include assessment of bone quality(12) and HO.(13) 
We have previously reported the use of Raman spectroscopy to identify changes in the extracellular matrix associated with $\mathrm{HO}(13-15)$ and calciphylaxis.(16) However, this technique is limited to the detection of suspicious calcification and does not focus on angiogenesis. Tools to image blood vessels would have utility not only for identifying changes associated with $\mathrm{HO}$, but also for evaluating other defects of angiogenesis related to poor wound healing.

\section{Materials and Methods}

Here we employ micro-CT, Raman spectroscopy, and reflectance spectroscopy to assess serial wound healing in a validated mouse model of heterotopic ossification. This study was approved by the University of Michigan Institutional Animal Care and Use Committee (IACUC; Protocol \# 00005909). Two experimental groups were evaluated - mice received either tendon transection alone $(\mathrm{n}=2)$ or dorsal burn with tendon transection $(\mathrm{n}=2)$ as previously described.(14) For all comparisons, the experimental group was the leg that underwent tendon transection and the control group was the contralateral leg. For the purposes of this study, tendon transection and dorsal burn with tendon transection were combined $(\mathrm{n}=4)$ as to compare injured versus uninjured tissue. Spectroscopy measurements were taken at five pre-specified sites along the injured and uninjured posterior hindlimb of each mouse (Fig 1a). Measurements were obtained immediately prior to surgery as baseline (T0), immediately following surgery (T1), 1 week after surgery (T2), 3 weeks after surgery (T3), 6 weeks after surgery (T4), or 9 weeks after surgery (T5). Microcomputed tomography (MicroCT) imaging was then performed to confirm the presence or absence of HO 9 weeks after injury. MicroCT imaging showed characteristic presence of radiographically evident $\mathrm{HO}$ corresponding to sites 1, 2, and 3 (Fig 1b). 
Spectra were collected with a hand-held filtered N-around-1 fiber optic probe (EMVision, Loxahatchee, FL; interrogating a tissue volume $<1 \mathrm{~mm}^{3}$ connected to a portable Raman spectroscopy system (Rxn 1, Kaiser Optical Systems; $785 \mathrm{~nm}$ laser) operated with 6-8 $\mathrm{cm}^{-1}$ resolution. The reflectance measurements were conducted using an unfiltered 1:1 probe connected to a portable spectrometer (USB2000+ coated for visible/near-infrared wavelengths, Ocean Optics; HL-2000-FHSA, Ocean Optics) operated with $<2$ nm resolution between 350-800 nm.

To produce high-quality spectra, the fiber probe system was operated with $60 \mathrm{sec}$ integration time for Raman spectra and $5 \mathrm{sec}$ integration time for reflectance spectra. In principle, measurement time could be reduced to 1-3 seconds for Raman and $<1$ second for reflectance collections. Such collection times could enable more thorough mapping, but scanning a relatively large region of interest remains difficult with a fiber-probe system. Spectra were preprocessed as previously described.(17) Prior to analysis, Raman spectra were normalized to the $1001 \mathrm{~cm}^{-1}$ phenylalanine band and reflectance spectra were normalized to their peak. Principal components analysis (PCA) was performed with built-in MATLAB ${ }^{\circledR}$ functions. Data was analyzed with a mixed linear effects model (SPSS $\left.{ }^{\circledR}\right)$ with experimental condition, measurement site, and measurement time point as fixed factors. A $p$-value $<0.05$ was deemed significant.

\section{Results}

Our preliminary results suggest that reflectance spectroscopy can be used to delineate vessel formation, and that pathologic wounds may be characterized by unique spectra (Fig. 2). In our model, HO formed at sites 1-3, while sites 4 and 5 did not have radiographic evidence of HO. In our experience, these findings are consistent with previous experiments we have 
performed using this HO model. Key features observed in the reflectance and Raman spectra principal components that warrant further study include: the slope of the reflectance curve at less than $525 \mathrm{~nm}$ and greater than $600 \mathrm{~nm}$, and the hemoglobin absorption bands;(10) the $\mathrm{CH}_{2}$ bending, Amide I and Amide III, and the proline and hydroxyproline Raman bands,(12) respectively.

\section{Discussion}

Wound healing follows a stereotypical progression beginning with initial confluence of hemostatic factors followed by inflammation, proliferation, and remodeling. The first two and final steps of hemostasis, inflammation, and remodeling are primarily non-angiogenic processes, reliant on agglutination of preformed platelets and fibrin along with recruitment and various modes of activation of cellular agents through chemotaxis and cytokine signaling. Proliferation in a normal setting exhibits a milieu of angiogenic cytokines including FGF, VEGF, TGF- $\beta$, and angiopoietin along with endothelial $\alpha v \beta 3$ integrin receptor for fibrin/fibronectin of the extracellular matrix induce capillary growth and supporting granulation tissue.(18) This av $\beta 3$ integrin receptor is particularly crucial in the gestalt of the proliferative step, reacting to the rich density of fibronectin from hemostasis. Various human diseases, inflammatory states, or any other process capable of disrupting this finely tuned recipe can lead to abnormally stunted or hyperactive neovascularization, augmenting the capacity to support important subsequent fibroblast and cellular infiltration, activity, and mechanical recovery central to healing. Many interventions prioritize minimization of systemic inflammation and endothelial damage: tight diabetic control, smoking cessation, and minimizing ischemia secondary to local pressure. Pressure ulcer management is one powerful illustration of less sophisticated yet effective 
management. Promising yet primarily theoretical sites for future investigation include strategies targeting biological pathways including activation or blockade of receptors for additional angiogenic molecules including CXCL4/8/12, angiostatin, PF4, and SDF-1 in addition to those discussed above.(19)

While preliminary, our results suggest that reflectance and Raman spectroscopy can be a useful multi-modal tool to assess an important component of the proliferative step of wound healing i.e. local angiogenesis. A key problem to be addressed for clinical translation of this dual-modal tool is developing a specialized hand-held fiber optic probe that is designed to detect angiogenesis in a broad range of complications, not limited to HO. Thus, the probe must be adaptable, requiring interrogation of multiple sites and depths to create a representative map of a sample rather than point sampling. Further, the probe will need to operate within varying tissue presentations, including but not limited to fatty tissue and pigmented skin. Once developed, this dual-modal tool is adjunct technology to pathology, CT imaging, and clinical standard of care.

\section{Conclusion}

In this work, we have demonstrated that dual-mode reflectance and Raman spectroscopy show promise to detect key features of both angiogenesis and mineralization adjacent to from the site of injury from the tissue surface. Future work will include development of an optimized design of the dual-mode instrument and more refined algorithms to characterize the extent of angiogenesis. 


\section{Acknowledgments}

Funding is gratefully acknowledged from NIH NIGMS K08GM109105-01, Plastic Surgery Foundation National Endowment Award, American Association of Plastic Surgery Academic Scholarship Award, DOD: W81XWH-14DMRDP-CRMRP-NMSIRA (to BL), NIH F32 AR066499 (to SA), Howard Hughes Medical Institute Fellowship (to SJL), and NIH R01 AR052010 (to MDM).

\section{References}

1.Board TN, Karva A, Board RE, Gambhir AK, Porter ML The prophylaxis and treatment of heterotopic ossification following lower limb arthroplasty. J Bone Joint Surg Br 2007:89:434440.

2.Vanden Bossche L, Vanderstraeten G Heterotopic ossification: a review. J Rehabil Med 2005:37:129-136.

3.Balboni TA, Gobezie R, Mamon HJ Heterotopic ossification: Pathophysiology, clinical features, and the role of radiotherapy for prophylaxis. Int $\mathrm{J}$ Radiat Oncol Biol Phys 2006:65:1289-1299.

4.Hoff P, Rakow A, Gaber T, Hahne M, Senturk U, et al. Preoperative irradiation for the prevention of heterotopic ossification induces local inflammation in humans. Bone 2013:55:93101.

5.Jens S, Koelemay MJ, Reekers JA, Bipat S Diagnostic performance of computed tomography angiography and contrast-enhanced magnetic resonance angiography in patients with critical limb ischaemia and intermittent claudication: systematic review and meta-analysis. Eur Radiol 2013:23:3104-3114.

6.Green NE, Chen SY, Messenger JC, Groves BM, Carroll JD Three-dimensional vascular angiography. Curr Probl Cardiol 2004:29:104-142.

7.Kedrin D, Gligorijevic B, Wyckoff J, Verkhusha VV, Condeelis J, et al. Intravital imaging of metastatic behavior through a mammary imaging window. Nat Methods 2008:5:1019-1021.

8.Boas D.A. PC, Ramanujam N. Handbook of biomedical optics. CRC Press 2011.

9.Lloyd WR, Wilson RH, Lee SY, Chandra M, McKenna B, et al. In vivo optical spectroscopy for improved detection of pancreatic adenocarcinoma: a feasibility study. Biomed Opt Express 2013:5:9-15.

10.Wilson RH, Chandra M, Chen LC, Lloyd WR, Scheiman J, et al. Photon-tissue interaction model enables quantitative optical analysis of human pancreatic tissues. Opt Express 2010:18:21612-21621.

11.Sujatha N. SABS, Bala Nivetha K., Narayanamurthy V.B., Seshadri P. Assessment of Microcirculatory Hemoglobin Levels in Normal and Diabetic Subjects using Diffuse Reflectance Spectroscopy in the Visible Region - a Pilot Study. Journal of Applied Spectroscopy 2015:82:432-437.

12.Morris MD, Mandair GS Raman assessment of bone quality. Clin Orthop Relat Res 2011:469:2160-2169.

13.Peterson JR, De La Rosa S, Sun H, Eboda O, Cilwa KE, et al. Burn injury enhances bone formation in heterotopic ossification model. Ann Surg 2014:259:993-998. 
14.Peterson JR, Eboda ON, Brownley RC, Cilwa KE, Pratt LE, et al. Effects of aging on osteogenic response and heterotopic ossification following burn injury in mice. Stem Cells Dev 2015:24:205-213.

15.Peterson JR, De La Rosa S, Eboda O, Cilwa KE, Agarwal S, et al. Treatment of heterotopic ossification through remote ATP hydrolysis. Sci Transl Med 2014:6:255ra132.

16.Lloyd WR, Agarwal S, Nigwekar SU, Esmonde-White K, Loder S, et al. Raman spectroscopy for label-free identification of calciphylaxis. J Biomed Opt 2015:20:80501.

17.Esmonde-White FW, Esmonde-White KA, Morris MD Minor distortions with major consequences: correcting distortions in imaging spectrographs. Appl Spectrosc 2011:65:85-98.

18.Tonnesen MG, Feng X, Clark RA Angiogenesis in wound healing. J Investig Dermatol Symp Proc 2000:5:40-46.

19.Bodnar RJ Chemokine Regulation of Angiogenesis During Wound Healing. Adv Wound Care (New Rochelle) 2015:4:641-650.

\section{Figure Legends}

Fig. 1 Study design, including (a) measurement sites and (b) micro-computed tomography results. Note that blue indicates heterotopic ossification, corresponding to anticipated sites of $\mathrm{HO}$ in (a).

Fig. 2 Raman and reflectance spectroscopy were analyzed with principal components analysis. Corresponding principal components were inputs into linear mixed effects model. Results demonstrate potential for spectroscopy to characterize experimental condition (Raman: PC1, reflectance: PC2), time point of measurement (Raman: PC2-3, reflectance: PC1-3), and site of measurement (Raman: PC3, reflectance: PC1-3). 
(a)
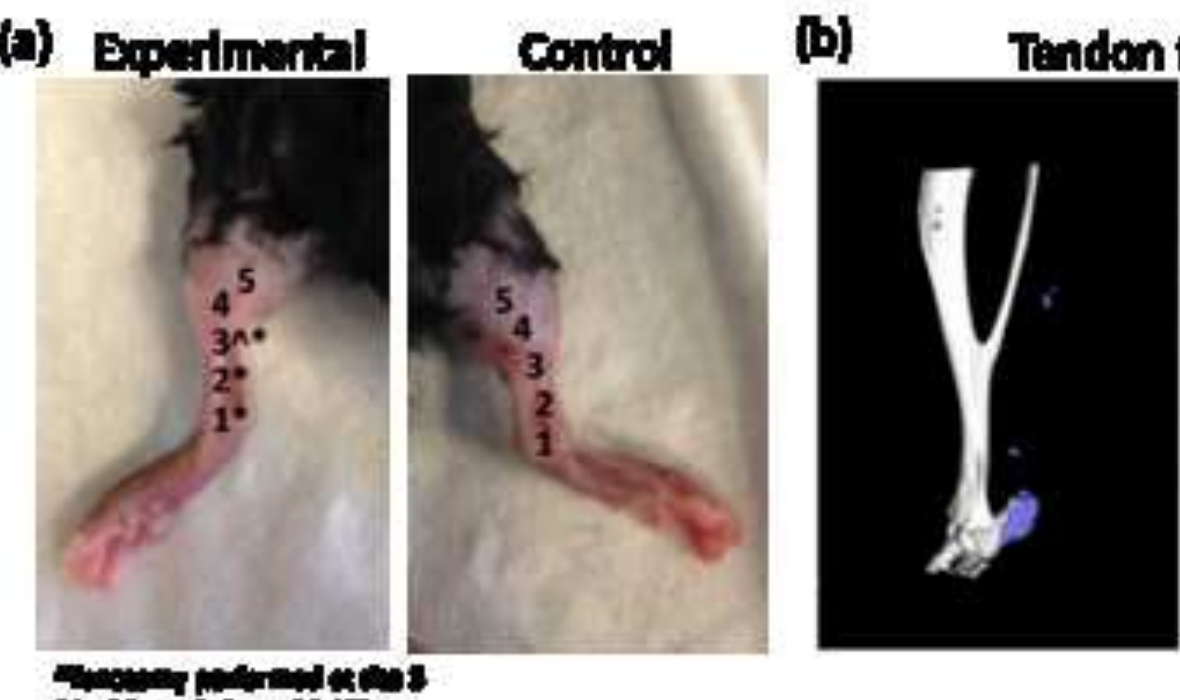

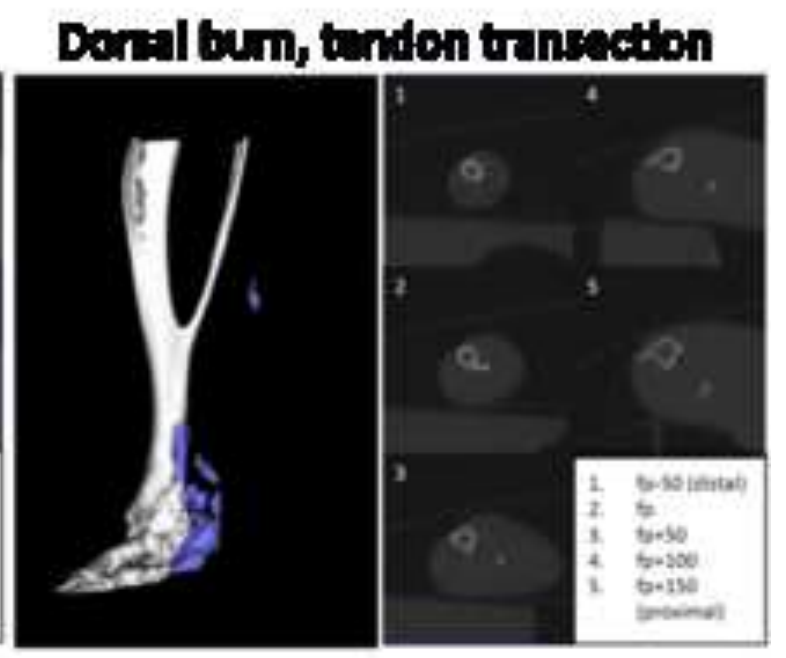

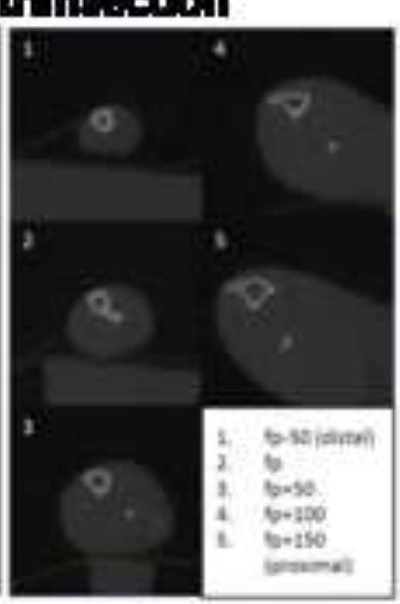

年 


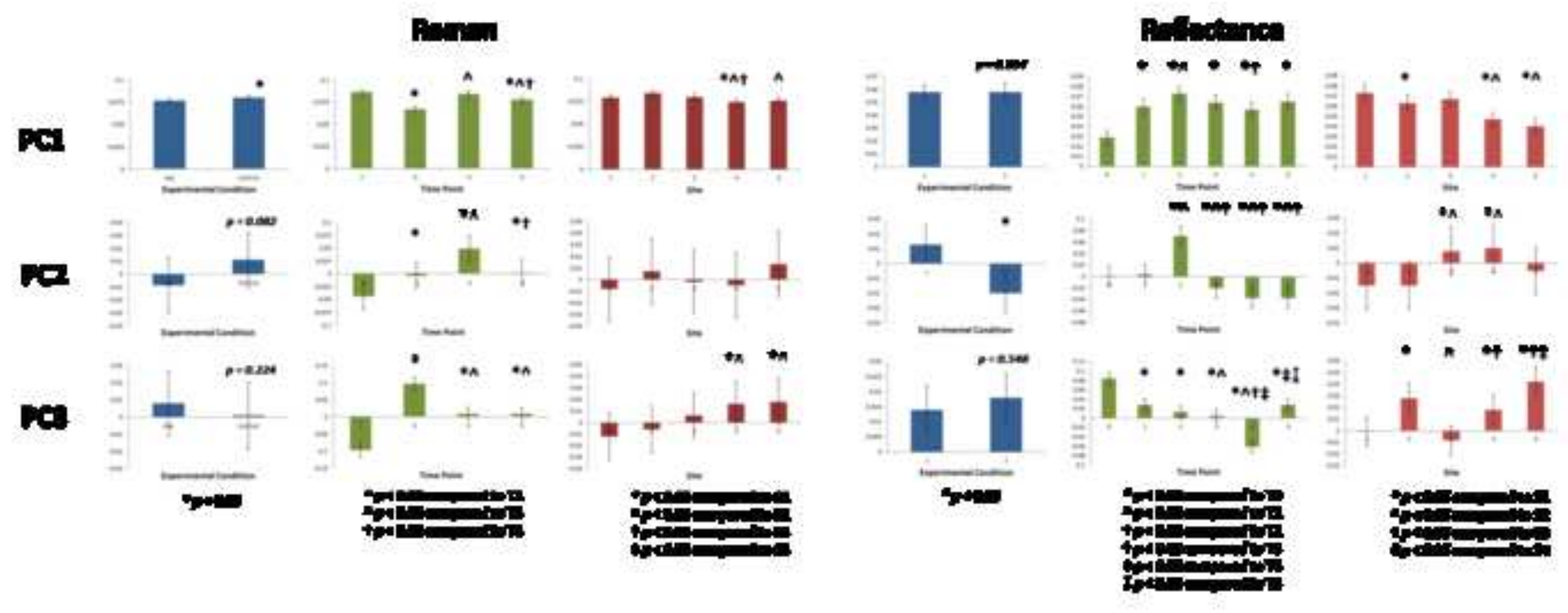

